\title{
Proposed Joint Propagation and Reinforcement Learning-based Television White Space Ledger
}

\author{
Armie E. Pakzad ${ }^{1}$, Abbas Ali Pakzad ${ }^{2}$, Lawrence Materum ${ }^{3}$ \\ ${ }^{1,3}$ Electronics and Communications Engineering Department, De La Salle University, 2401 Taft Ave., Manila, Philippines \\ ${ }^{2}$ College of Computer Studies, Arellano University, 3058 Taft Ave., Pasay Philippines \\ ${ }^{3}$ Tokyo City University, 1-28-1 Tamazutsumi, Setagaya, Tokyo, 158-8557, Japan \\ armie.pakzad@dlsu.edu.ph
}

\begin{abstract}
Television white spaces (TVWSs) are vacant television (TV) channels allocated to TV broadcasting. The usability of TVWS can be classified in terms of frequency, time, and location. One way of implementing TVWS communications is by using the TVWS database (TVWSDB). The purpose of this database is to secure the primary users (PUs) from interference from secondary users (SUs). Existing TVWSDBs do not have a prediction feature that provides short-term, medium-term, and long-term forecast data for secondary TVWS users, that could be useful for government and industry stakeholders. This paper proposes an improved TVWSDB that incorporates the reinforcement learning (RL) technique providing short-term, medium-term, and long-term forecast data on the available channels at a given location, time, and frequency for secondary TVWS users. RL is to be used to provide the prediction feature of the TVWSDB. The prediction feature is seen to lessen the number of queries per transaction instance and the search duration for the availability of channels would be lessened and is seen to be beneficial to TVWS users.
\end{abstract}

Keywords: Radio Propagation, Radio Spectrum Management, Reinforcement Learning, Television White Space Database.

\section{INTRODUCTION}

The emergence of mobile communications led to the exploitation of spectrum bands. In contrast, the utilization of other bands is minimal, specifically, the TVWS which refers to the unused portions of spectrum in the very high frequency (VHF) to ultra-high frequency (UHF) bands. The immense shift from analog to digital TV is becoming the solution to spectrum scarcity brought by the fast-rising adoption of mobile communications. Cognitive radio (CR) and software-defined radio (SDR) are seen to be solutions to more effective use of the spectrum[1].Hence, resolving the issue of spectrum insufficiency is the main objective of CR. It detects its immediate environment, traces the changes, and responds to its results [2]. SDR is a platform that can be used to utilize the unused channels for broadcasting which is known as TVWS. TVWS is a distinguished new opportunity for wireless communications due to its capabilities of penetrating obstacles as well as its low utilization at given times and specific areas. Obstacles like buildings or big terrains are mainly the cause of radio wave propagation loss[3], this makes TVWS a better option for wireless communications since it can penetrate these obstructions. Hence, it can successfully enhance the effective use of spectrum and resolve the worldwide problem in spectrum scarcity [4]. The use of TVWS communications is seen to improve the delivery of services in rural areas. Only 15\% of the TV spectrum is utilized at present, thus it could be a great way to provide connectivity to almost 3 billion people all over the world[5].

The spectrum management is imperative for efficient wireless communication services. Policies and standards may change over time since TVWS is considered to be one of the solutions to the growing needs in the spectrum utilization. Adjustments on the part of the industry should be made in case of policy amendments. These modifications should be done immediately so as not to interrupt the delivery of services. Furthermore, it should not be costly on the part of the industry to avoid extra charges on the part of the consumers.

Scalable SDR can address the above scenario in case of policy changes on the use of the spectrum.TVWS deployment is one promising solution to the growing needs in wireless communications. Wireless communication is rapidly growing and the demand for more efficient spectrum management is rising. Database-assisted TVWS technology is likely one of the suggested methods to deal with this demand. The currently released television (TV)spectrum brought by the migration to digital TV can offer nearly an additional $300 \mathrm{MHz}$ of spectrum bandwidth. At first, TVWS technology was intended to be carried on by spectrum sensing algorithms (SSA).Despite this, some test results from the SSA were unacceptable. Thus, spectrum regulatory groups have started the mandate in the use of TVWS technology with geolocation technology. There are three parameters to consider to implement a geolocation database for the availability of 
Armie E. Pakzad et al., International Journal of Emerging Trends in Engineering Research, 8(4), April 2020,1450 - 1456

TVWS inside the topographical area, this includes frequency dimensions (3D), time, and location[6].Since in some cases, there is no satisfactory assurance for wireless systems operating in similar frequency bands to be interference-free, the utilization of databases for more effective spectrum management was recommended. Undesirable circumstances like recurrent channel switching can be avoided once the frequency allocation is regulated. This would improve the quality of service in the operations of wireless systems[7].

Based on the related works and to the best of the authors' knowledge, the existing TVWSDBs is unclear to have artificial intelligence (AI) that provides prediction feature which offers short-term, medium-term, and long-term forecast data for secondary TVWS users that is useful for government and industry players, other than that, existing TVWS radios are set in hardware than software. If there will be changes in government policies and industry or research on TVWS, then existing solutions will not be able to cope up.

This paper proposes an improved TVWSDB with a graphical user interface, that incorporates the reinforcement learning (RL), technique, a subsystem of machine learning (ML). ML methods assist in straightforward and swift inference for analysis [8].RL provides short-term, medium-term, and long-term forecast data on the available channels at a given location, time, and frequency for secondary TVWS users. RL will be used to provide artificial intelligence (AI) for the TVWSDB. It is a subsystem of machine learning which is focused on learning what is to be done based on the mapping of situations to actions to get the maximum reward. With the use of the RL algorithm, the proposed AI-based TVWSDB would be able to provide prediction data to TVWS operators (TVWSO) and other stakeholders. The prediction feature is seen to lessen the number of queries per transaction instance and the search duration for the availability of channels would be lessened that is seen to be beneficial to TVWS users.

This paper is organized as follows: Section 2 presents the previous works which are significant to this study; Section 3 expounds the novelty of the study by discussing the lacking in the approaches of the previous studies; Section 4 presents the methodology which includes the theoretical considerations that can be useful in designing the proposed AI-based TVWSDB, it also presents the block diagram of the proposed design, and Section 5 discusses the conclusion and future work.

\section{RELATED STUDIES}

This section discusses several studies conducted on the utilization of TVWS for communications. The following were the significant works related to this study.

The focus of [9] is on one of the functions of the CR network (CRN), which is spectrum mobility (SM). It described CRN as a method that can be used to take advantage of the unlicensed spectrum bands and that $\mathrm{CRN}$ has four main functions such as spectrum mobility, spectrum sharing, spectrum decision, and spectrum sensing. SM is also known as a handoff that supervises the movements of secondary users (SUs) to occupy the vacant unlicensed spectrum without interfering with the primary users' (PUs) transmission. The proponents presented a fuzzy logic approach consisted of two Fuzzy Logic Controllers for spectrum mobility in CRN. In this paper, price and duration were considered two main input parameters. With the said approach, the handoff delay will be decreased by $46-62 \%$ provided that the SU properly chooses the suitable PU.

The study discussed in [10]focused on the combination of a web-based geolocation database and wireless microphone sensors. With this method, the SUs will avoid interference with the PUs. The experimental results showed that the platform was able to provide an updated record of vacant channels using the geolocation database with the help of the information offered by the blind sensing algorithms.

The authors of [8]proposed a real-time application of a Cooperative Spectrum Sensing (CSS) testbed with the use of a front-end tool utilizing a database Fusion Center. SDR equipment was evaluated in a real-time application for the Brazilian digital TV set-up. The implementation of a database fusion center aided in achieving detection, misdetection, and false alarm rates for various signals and the rate of noise received[11].

In [12], the researchers evaluated the spectrum sensing and Federal Communications Commission (FCC) geolocation TVWS and compared the two methods. Both spectrum sensing and geolocation parameters were converted to field strength to compare the two based on the protection contour's physical space, the spectrum sensing's contour plus the geolocation's contour. Results showed that the channel discovery by the Advanced Television Systems Committee (ATSC) signal using a spectrum sensor, external of the protection contour was classified by the FCC as false alarms, however, detection within the spectrum sensing contour was found out to be true detection.

The authors of[6]presented a literature survey on recent developments and research directions on TVWS. The researchers discussed the architecture and operations of database assisted TVWS as well as its economic framework. The paper highlighted the operations of a database assisted TVWS network architecture as seen in Figure 1.Initially, a master TVWSD inquires to the spectrum regulator for the authorized TVWSDB operators that have passed through a qualifying procedure governed by the spectrum regulator. This process includes the examination of TVWSDB operators to assure the users that their databases operate based on the particular terms and conditions to operate. Second, the spectrum regulatory responds to the master TVWSD by providing a directory listing of authorized TVWSDB operators. Subsequently, the master TVWSD inquires to the 
selected authorized TVWSDB operators for vacant TVWS channels in its current site by specifying its operating parameters which consist of antenna information concerning the Height Above Average Terrain (HAAT), the frequency range, and the maximum transmission power. A TVWSDB then performs the required computations based on the operating parameters sent by the TVWSD in a query. The spectrum regulator furnishes the TVWSO with a database specifying the spectrum utilization for Digital Terrestrial Television (DTT), registered PMSE, and wireless microphones. TVWSO replies to the TVWSD by sending information on the available TVWS bands and the transmission power to be used. Thereafter, a slave TVWSD inquires to the master TVWSD on the transmission parameters and TVWS frequency to be used by providing its identifier called the TVWSD's MAC identity. The slave TVWSD may either ask for TVWS specific operational parameters or rely on generic parameters. Finally, the master TVWSD replies to the slave TVWSD by broadcasting the generic operating parameters. Slave TVWSD listens to the broadcast before transmission and decodes the generic operational parameter information. In case of a request for operating parameters, the master TVWSD passes on this data to the TVWSDB that performs the calculation of the optimum parameters for the specific slave device.

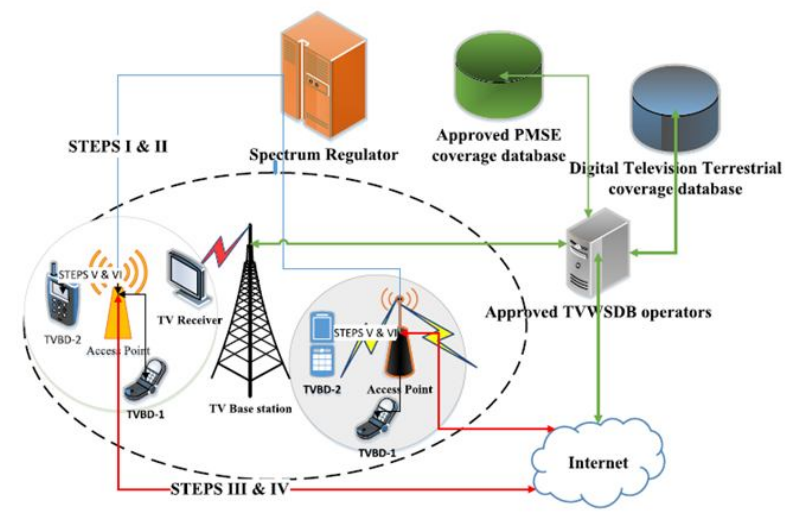

Figure 1: Database-assisted TVWS Network Architecture[6]

Based on the survey results, it was presumed that TVWS technology is socially recognized, which is based on the global pilot testing done successfully. From an economic point of view, an affordable model for the secondary spectrum market should be planned and designed. From the technical standpoint, TVWS technology using TVWSDB, issues about self-coexistence, and heterogeneous coexistence should be considered in implementing the said technology[6].

The proponents in [13]presented an analysis of the CR and TVWS for the implementation of a geolocation database that was focused on the detection of available TVWS channels which will protect the PUs. Different geolocation database architectures were discussed and used as a guide in developing their database. The web interface was provided to allow public access. Java programming language with the Spring framework was used to design the TVWSDB.
The previous study discussed in[14]provided an overview of several outcomes of the initial measurements on the available spectrum performed in Veralum, Durban South Africa in February 2016. The results were compared to that of the Council for Scientific and Industrial Research (CSIR)geolocation database (GD). Based on the results, there were discrepancies in the data provided by the geolocation database and the measured one. Slight discrepancies were observed when measurements were done in higher spots in locations with minor obstruction. On the other hand, the discrepancies became higher with measurements done in lower spots, in locations with higher obstructions. It was observed that the geolocation database performance could be better if real-time feedback will be implemented for a more precise forecast.

The researchers in [15]proposed a Dynamic Database Agent Middleware which serves as an interface between the SUs and GD. The middleware synchronizes with the GD in selecting the vacant channels to be used by the SUs. It uses the data based on the coming SUs from any topographical site and the projected vacant channel transmission time of each SU. The probable collisions between SUs were determined statistically. Based on the results, the proposed middleware decreases the collisions by $52.3 \%$.

\section{LACKING IN THE APPROACHES}

TVWSDB is an essential component of architecture design for the utilization of TVWS. Studies on TVWSDB included in the previous section did not explicitly mention the prediction using AI with the RL algorithm, on the operations of the databases. Also, to the best of the authors' knowledge, there was no discussion on the TVWSDB that offers short-term, medium-term, and long-term forecast data for secondary TVWS users. Geolocation databases included in the preceding studies did not mention the algorithm used for the intelligence of the GD. None of the studies explicitly stated about AI-based GD particularly using RL on searching for the availability of channels and did not include speed or search duration as a metric of success. This paper proposes an improved TVWSDB with a graphical user interface, that incorporates the reinforcement learning (RL) technique providing short-term, medium-term, and long-term forecast data on the available channels at a given location, time, and frequency for secondary TVWS users. RL will be used to provide artificial intelligence (AI) for the TVWSDB. With the use of the RL algorithm, the proposed AI-based TVWSDB would be able to provide prediction data to TVWS operators (TVWSO) and other stakeholders. The prediction feature is seen to lessen the number of queries per transaction instance and the search duration for the availability of channels would be lessened that would be beneficial to TVWS users 
Armie E. Pakzad et al., International Journal of Emerging Trends in Engineering Research, 8(4), April 2020,1450 - 1456

\section{METHODOLOGY}

This section discussed the theoretical considerations that can be useful in designing the proposed AI-based TVWSDB. It also presents the block diagram of the proposed design.

\subsection{Spectrum Manager}

Figure 2 shows the diagram of the spectrum manager (SM)that comprises logical interfaces namely, Customer Premises Equipment (CPE) Registration and Tracking, Channel Set Management, Policies, Geolocation, Self-Coexistence, Incumbent Database Service, and Spectrum Sensing Automation. SM is like the brain of Wireless Regional Access Network (WRAN)Base Station (BS), shown in the cognitive plane IEEE 802.22 BS reference model. SM controls decisions in the WRAN cells for the vacant spectrum and consumption to confirm the protection of incumbents and effective usage of the spectrum while conforming with the regulations. BS and CPE both include entities called Spectrum Sensing Algorithm (SSA) which interface with Spectrum Sensing Function (SSF) and that transmits sensing process-based SM command.

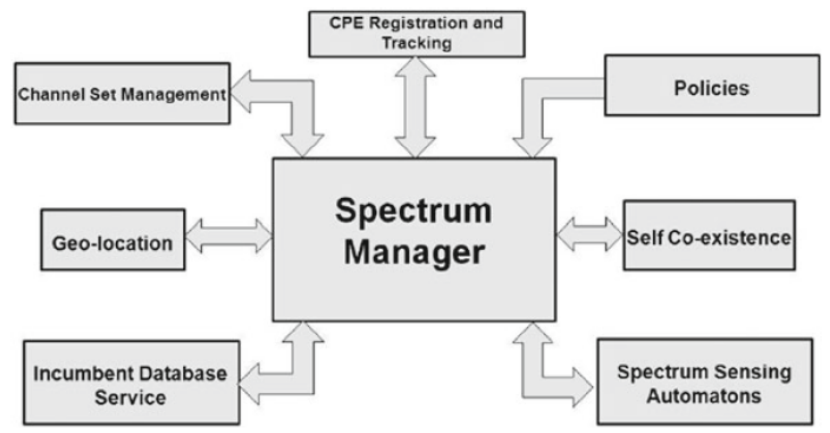

Figure 2: Spectrum Manager [16]

SM can perform the following functions; (i) maintain spectrum availability information and (ii) channel classification, and selection based on availability[16].

\subsection{Geolocation Technology}

Geolocation is a technology used in locating the place of a device within a network. There are two approaches in geolocation, the satellite-based geolocation, and terrestrial-based location. The previous is mandatory while the latter is supported by code division multiple access (CDMA). The satellite-based geolocation antenna is used in determining the longitude and latitude of the antenna in operation inside a 50-meter radius. On the other hand, terrestrial geolocation encompasses a process that includes two steps. The first one determines the scope with great accuracy and then uses a triangulation technique to determine the geolocation of CPE [16].

\subsection{TVWS Database (TVWSDB)}

A TVWSDB or a geolocation database refers to a central server that logs the data of all primary users' Primary User
(PU) locations and protection contours to provide frequency availability also known as TVWS at different locations. The TVWSDB is periodically updated. Before transmission, the TVWSDs look up the database through an interface to learn the available frequencies at its position. The TVWSDB calculates the frequency availability based on PUs' protection contours.

Figure 3 shows the protection region, protection contour, separation distance, and keep-out contour of a PU. The TVWSDB creates a protection region around a PU based on its operating information. The protection region is the coverage of the PUs transmitter. A separation distance from the boundary of the protection region is used to form a keep-out contour, this is done to protect the PU's receivers within the protection region. A channel that is located outside the keep-out contour is the one available to the Secondary User (SU). The TVWSDB determines the location of SU whether it is inside or outside the PUs' keep-out contours, then notifies the SU of the available channels. An enhanced TVWSDB is capable of providing additional information on the quality of white space based on the number of active TVWSDs and their powers in the related area. it requires feedback from the TVWSD which includes location, transmission frequency, and power, RF bandwidth, and other parameters[17].



Figure 3: Protection Region, Protection Contour, Separation Distance, and Keep-out Contourof a PU[17]

\subsection{Reinforcement Learning}

Reinforcement learning (RL) is a subsystem of machine learning which is focused on learning what is to be done based on the mapping of situations to actions to get the maximum reward. The learner learns by trying which actions must be taken to get the reward instead of being told what to do. In some tough situations, actions may affect the direct reward and perhaps the succeeding rewards[18].Two significant features of RL are trial-and-error search, and delayed reward. RL operates on a dynamic data set from the environment. Learning from a dynamic environment means to realize the best actions that will create the optimum result. The agent learns, discovers, and works with the environment. First, the agent should examine the present condition of the environment. Based on its observation, it chooses the appropriate action to be taken. The environment then switches to another condition and generates a reward for that action which will be obtained by the agent. Based on the latest data, the agent can ascertain if the action was good 
enough to be repeated or bad to be avoided. The observation-action-reward cycle takes up again until learning is accomplished[19].

Figure 4shows the anatomy of RL. A function can be found inside the agent's frame which accepts observations as inputs and maps it to actions that can be depicted as the outputs. The function is the one consists of elements of the control system. the function is referred to as the policy which determines the appropriate action to be taken based on the observations. When the actions taken were good, the environment generates a reward. However, since the environment is said to be dynamic, it may be altered so static mapping may not be the best. For this reason, the RL algorithm is introduced as depicted in Figure 5. The RL algorithm updates the policy based on the selected actions, environmental observations, and the gathered rewards. The RL algorithms will be used by the agent to learn the optimal policy during its interaction with the environment to take the best action at any given situation, that is, the action that will make the highest reward in time[19].



Figure 5: Anatomy of Reinforcement Learning[19]

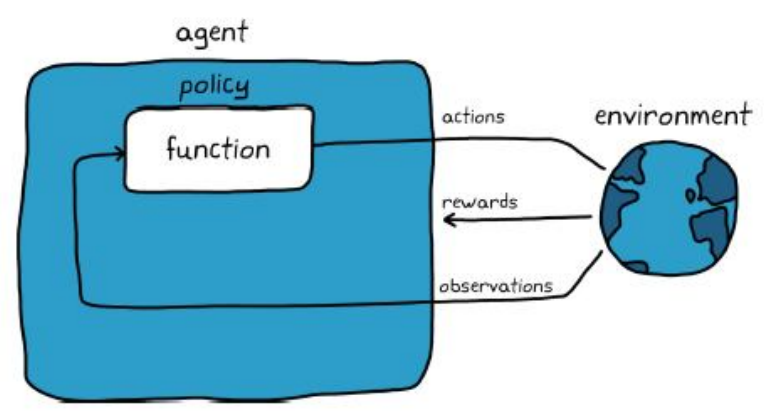

Figure 6: Learning the Optimal Policy[19]

\subsection{Diagram of the Proposed AI-Based TVWSDB}

The function of the AI-based TVWSDB would be simulated using MATLAB. Figure 6 outlines the methodology of the study. TVWSO will be represented by individual computers with a Graphical User Interface (GUI) in which the required WSD data will be entered. These data will be used to inquire about which available channels are suitable for the inquiry, based on its location, frequency, and time. These data will be processed by the AI-based TVWS predictor, a part of the TVWSDB, represented by a GUI which will display the list of available channels for the inquiring WSD. The proposed AI-based TVWS predictor will perform the calculations according to the stored data in the TVWSDB. The TVWSDB is a database that stores the necessary information to find out the available channels in a location immediately. It contains the following information; (i) TV tower data, associated with the registered primary TV towers or other PUs, (ii)other licensed user data such as wireless microphones, (iii) WSD data that are associated to unlicensed secondary WSDs, and (iv) regulatory data which refers to the required parameters which are determined and designated are established and indicated by a regulatory body[13]. Since TVWS technology is not implemented yet in the Philippines, the study will use the data defined in [13]. When a WSD or TVWSD occupies a channel, it is supposed to be listed to the database to record that the channel is taken and is no longer available at an instant. The TVWS predictor incorporates the reinforcement learning (RL) technique providing short-term, medium-term, and long-term forecast data on the available channels at a given location, time, and frequency for secondary TVWS users. RL will be used to provide artificial intelligence (AI) for the TVWSDB's predictor. With the use of the RL algorithm, the proposed AI-based TVWS predictor as part of the TVWSDB would be able to provide prediction data to TVWS operators (TVWSO) and other stakeholders. The prediction feature is seen to lessen the number of queries per transaction instance and the search duration for the availability of channels would be lessened that is seen to be beneficial to TVWS users.

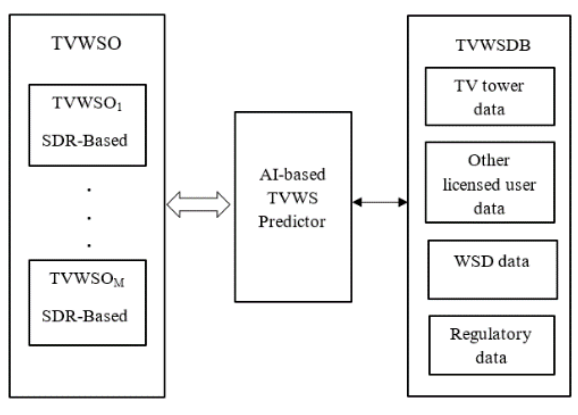

Figure 6: Diagram of the Proposed AI-Based TVWSDB

\subsection{Parameters of the Proposed TVWSDB}

The contents of the proposed TVWSDB are listed in Table 1 and Table 2. The TVWSDB parameters used by the Canadian government [20] were considered along with those specified in [13]. The parameters were itemized under three categories: (i) TV tower, (ii) other licensed users, and (iii) TVWSD data. The TV tower information consists of data associated with the registered TV stations and other PUs which mainly consist of transmit power, transmit and receive frequencies, antenna height, site elevation, transmitter antenna angle elevation, and other data on the TV station. Other registered users such as wireless microphone may be existing aside from the licensed 
Armie E. Pakzad et al., International Journal of Emerging Trends in Engineering Research, 8(4), April 2020,1450 - 1456

towers. In this case, it must be listed in the TVWSDB in order to guarantee the correct process. TVWSD data includes the device type, its ID, location and transmission channel.

Table 1: Initial Parameters of the Proposed TVWSDB[13],[20]

\begin{tabular}{|c|c|c|}
\hline $\begin{array}{c}\text { Data } \\
\text { Category }\end{array}$ & Elements & Description \\
\hline \multirow{18}{*}{ TV Tower } & Tower ID & $\begin{array}{l}\text { Identification of the } \\
\text { transmitting tower }\end{array}$ \\
\hline & $\begin{array}{l}\text { Transmitter } \\
\text { Type }\end{array}$ & $\begin{array}{l}\text { To specify the type of } \\
\text { tower transmitter }\end{array}$ \\
\hline & Tower Location & Province, City \\
\hline & Transmit Power & $\begin{array}{l}\text { The tower's transmit } \\
\text { power }\end{array}$ \\
\hline & $\begin{array}{l}\text { Transmit } \\
\text { Frequency } \\
(\mathrm{MHz})\end{array}$ & $\begin{array}{l}\text { Centre frequency of the } \\
\text { transmit channel }\end{array}$ \\
\hline & $\begin{array}{l}\text { Receive } \\
\text { Frequency } \\
(\mathrm{MHz})\end{array}$ & $\begin{array}{l}\text { Centre frequency of the } \\
\text { receive channel }\end{array}$ \\
\hline & Transmit Lower & The upper band $(\mathrm{MHz})$ \\
\hline & Transmit Upper & The lower band (MHz) \\
\hline & Antenna Height & $\begin{array}{c}\text { Tower's height above the } \\
\text { terrain (HATT) }\end{array}$ \\
\hline & Latitude & $\begin{array}{l}\text { Latitude (north) of the } \\
\text { station }\end{array}$ \\
\hline & Longitude & $\begin{array}{l}\text { Longitude (west) of the } \\
\text { station }\end{array}$ \\
\hline & $\begin{array}{l}\text { Site Elevation } \\
(\mathrm{m})\end{array}$ & $\begin{array}{c}\text { Elevation of the site's } \\
\text { ground level above mean } \\
\text { sea level }\end{array}$ \\
\hline & $\begin{array}{l}\text { Structural } \\
\text { Height (m) }\end{array}$ & $\begin{array}{l}\text { Total height above } \\
\text { ground level the structure } \\
\text { on which the antennas are } \\
\text { mounted }\end{array}$ \\
\hline & $\begin{array}{c}\text { Transmitter } \\
\text { Antenna } \\
\text { Elevation Angle }\end{array}$ & $\begin{array}{l}\text { Transmitter antenna's } \\
\text { vertical elevation angle } \\
\text { of the main lobe with } \\
\text { reference to the } \\
\text { horizontal ground in } \\
\text { degrees }\end{array}$ \\
\hline & $\begin{array}{c}\text { Transmitter } \\
\text { Antenna Gain }\end{array}$ & $\begin{array}{l}\text { Gain of the transmitter } \\
\text { antenna expressed } \\
\text { relative to an isotropic } \\
\text { radiator }(\mathrm{dBi}) .\end{array}$ \\
\hline & $\begin{array}{l}\text { Transmitter } \\
\text { Antenna } \\
\text { Directional }\end{array}$ & $\begin{array}{c}\text { Identifies if the } \\
\text { transmitter antenna is } \\
\text { Omni directional } \\
\text { (radiation pattern of } 360 \\
\text { deg.) or directional. }\end{array}$ \\
\hline & $\begin{array}{l}\text { Last Date } \\
\text { Modified }\end{array}$ & $\begin{array}{l}\text { The date on which the } \\
\text { station or associated } \\
\text { channels were modified } \\
\text { or operational }\end{array}$ \\
\hline & $\begin{array}{l}\text { Last Upload } \\
\text { Date. }\end{array}$ & $\begin{array}{l}\text { Indicates if there have } \\
\text { been any changes to the } \\
\text { station or channels since } \\
\text { the last upload. }\end{array}$ \\
\hline
\end{tabular}

Table 2: Initial Parameters of the Proposed TVWSDB[13],[20](Continuation of Table 1)

\begin{tabular}{|c|c|c|}
\hline $\begin{array}{c}\text { Data } \\
\text { Category }\end{array}$ & Elements & Description \\
\hline \multirow{4}{*}{$\begin{array}{l}\text { Other } \\
\text { Licensed } \\
\text { Users }\end{array}$} & User type & $\begin{array}{l}\text { PMSE e.g. } \\
\text { microphone }\end{array}$ \\
\hline & User ID & $\begin{array}{c}\text { Registered user's } \\
\text { identification }\end{array}$ \\
\hline & Device's location & $\begin{array}{l}\text { Latitude and } \\
\text { longitude }\end{array}$ \\
\hline & $\begin{array}{c}\text { Transmission } \\
\text { Channel }\end{array}$ & $\begin{array}{c}\text { The channel used } \\
\text { by the device }\end{array}$ \\
\hline \multirow{5}{*}{ TVWSD } & Device type & $\begin{array}{l}\text { Identifies if the } \\
\text { TVWSD is either } \\
\text { secured or } \\
\text { individual }\end{array}$ \\
\hline & TVWSDID & $\begin{array}{l}\text { TTVWSD } \\
\text { identification }\end{array}$ \\
\hline & $\begin{array}{l}\text { TVWSD serial } \\
\text { number }\end{array}$ & $\begin{array}{c}\text { Used to } \\
\text { authenticate the } \\
\text { TVWSDB }\end{array}$ \\
\hline & $\begin{array}{l}\text { TVWSD's } \\
\text { location }\end{array}$ & $\begin{array}{c}\text { The position of } \\
\text { the TVWSD }\end{array}$ \\
\hline & $\begin{array}{c}\text { Transmission } \\
\text { channel }\end{array}$ & $\begin{array}{l}\text { TVWSD's current } \\
\text { transmission } \\
\text { channel }\end{array}$ \\
\hline
\end{tabular}

\section{CONCLUSION AND FUTURE WORK}

TVWS technology is a promising solution to spectrum scarcity, with its superior propagation characteristics, it is seen to solve connectivity problems in rural areas. Existing studies on geolocation databases included in the literature survey on TVWSDBs did not have the prediction feature using AI incorporating the RL method, on the operations of the databases. This paper proposes an AI-based TVWSDB for short-term, medium-term, and long-term forecast data for secondary TVWS users. This study is geared towards an improved TVWSDB with a prediction feature that would minimize the search duration for the channel availability for SUs. Future work will focus on the performance comparison between the non-AI-based TVWSDB and the initial design of the AI-based TVWSDB in terms of the search duration or speed.

\section{ACKNOWLEDGMENT}

The authors acknowledge De La Salle University for its support for the publication of this paper. 
Armie E. Pakzad et al., International Journal of Emerging Trends in Engineering Research, 8(4), April 2020,1450 - 1456

\section{REFERENCES}

[1] A. Prata, A. S. R. Oliveira, and N. B. Carvalho, "An Agile Digital Radio System for UHF White Spaces," IEEE Microw. Mag., vol. 15, pp. 92-97, 2014. https://doi.org/10.1109/MMM.2013.2288809

[2] C. Kocks, A. Viessmann, G. H. Bruck, and P. Jung, "Concept design and performance evaluation of a parametrizable cognitive radio engine," in 2012 IEEE Global Communications Conference (GLOBECOM), 2012, pp. 1109-1113.

[3] A. D. Africa, L. R. Bulda, E. Del Rosario, M. Z. Marasigan, and I. N. Navarro Isabel Matthew Zandrick Marasigan, "Radio Wave Propagation: Simulation of Free Space Propagation Path Loss," Int. J. Emerg. Trends Eng. Res., 2020. https://doi.org/10.30534/ijeter/2020/07822020

[4] Y. Luo, L. Gao, and J. Huang, "Business modeling for TV white space networks," IEEE Commun. Mag., vol. 53, pp. 82-88, 2015.

[5] E. Trinidad and L. Materum, "Juxtaposition of Extant TV White Space Technologies for Long-Range Opportunistic Wireless Communications," Int. J. Emerg. Trends Eng. Res., 2019. https://doi.org/10.30534/ijeter/2019/17782019

[6] K. H. Anabi, R. Nordin, and N. F. Abdullah, "Database-assisted television white space technology: challenges, trends and future research directions," IEEE Access, vol. 4, pp. 8162-8183, 2016.

[7] M. Höyhtyä, J. Ylitalo, X. Chen, and A. Mämmelä, 'Use of databases for dynamic spectrum management in cognitive satellite systems," in Cooperative and Cognitive Satellite Systems, Elsevier, 2015, pp. 453-480.

[8] A. Vijaya Lakshmi, K. V. T. Nagendra Babu, M. Sree Ram Deepak, A. Sai Kumar, V. Chandra Sekhar Yadav G. V. P. Gopi Tilak, and V. S. Ghali, "A Machine Learning based Approach for Defect Detection and Characterization in Non-Linear Frequency Modulated Thermal Wave Imaging," Int. J. Emerg. Trends Eng. Res., 2019.

[9] A. Alhammadi, M. Roslee, and M. Y. Alias, "Fuzzy logic based negotiation approach for spectrum handoff in cognitive radio network," in 2016 IEEE 3rd International Symposium on Telecommunication Technologies (ISTT), 2016, pp. 120-124. https://doi.org/10.1109/ISTT.2016.7918097

[10] R. Dionísio, J. Ribeiro, J. Ribeiro, P. Marques, and J. Rodriguez, "Cross-platform demonstrator combining spectrum sensing and a geo-location database," in 2012 Future Network \& Mobile Summit (FutureNetw), 2012, pp. 1-9.

[11] J. J. A. Arnez, L. S. Mello, C. Rodriguez, C. A. Medina, and P. Gonzalez, "Real Time SDR Cognitive Radio System for Cooperative Spectrum Sensing in the $\mathbf{7 0 0}$ MHz Brazilian Digital TV Band," in 2018 IEEE-APS Topical Conference on Antennas and Propagation in Wireless Communications (APWC), 2018, pp. 791-794.

[12] S. J. Shellhammer, "A comparison of geo-location and spectrum sensing in cognitive radio," in 2009
Proceedings of 18th International Conference on Computer Communications and Networks, 2009, pp. $1-6$.

[13] N. Zurutuza, "Cognitive Radio and TV White Space Communications: TV White Space Geo-location Database System," Master's Thesis, Institutt for elektronikk og telekommunikasjon, 2011.

[14] A. A. Lysko and L. Mfupe, "Geolocation Spectrum Database - Based TV Spectrum Availability Predictions versus Measurements for Verulam, South Africa: Overview," in Proc. 13th European Conf. Antennas and Propagation (EuCAP), Mar. 2019, pp. 1-5.

[15] G. Atta-Boateng, P. O. Bobbie, K. O. Boateng, and E. K. Akowuah, "Reducing secondary user collisions in TV White Space through a Geolocation Database Middleware," in 2018 Sixth International Conference on Digital Information, Networking, and Wireless Communications (DINWC), 2018, pp. 18-27. https://doi.org/10.1109/DINWC.2018.8356989

[16] A. K. Mishra and D. L. Johnson, White Space Communication: advances, developments and engineering challenges. Springer, 2014. https://doi.org/10.1007/978-3-319-08747-4

[17] S. W. Oh, Y. Ma, M.-H. Tao, and E. Peh, TV white space: The first step towards better utilization of frequency spectrum. John Wiley \& Sons, 2016.

[18] R. S. Sutton and A. G. Barto, Reinforcement learning: An introduction. MIT press, 2018.

[19] Mathworks, "Reinforcement Learning with MATLAB Understanding the Basics and Setting Up the Environment the Environment." available at https://www.mathworks.com/content/dam/mathworks/e book/gated/reinforcement-learning-ebook-part1.pdf

[20] Government of Canada, DBS-01 - White Space Database Specifications. 2020, available at https://www.ic.gc.ca/eic/site/smt-gst.nsf/eng/sf10928.ht $\mathrm{ml}$ 